\title{
The illusion of a chest wall tumor: a case report of sternal tuberculosis
}

\author{
Morgan Daniel $^{1}$, Charles Ricordel ${ }^{2}$, Aurélien Lorleac'h ${ }^{3}$, James Norwood ${ }^{4}$, Bertrand Richard De Latour ${ }^{5}$, \\ Simon Rouzé 6 , Jean-Philippe Verhoye ${ }^{7}$ \\ ${ }^{1}$ Division of Thoracic and Cardiovascular Surgery, University Hospital of Rennes; ${ }^{2}$ Division of Pneumology, University \\ Hospital of Rennes; ${ }^{3}$ Division of Internal Medicine and Infectious Diseases, Hospital of Lorient; ${ }^{4}$ Division of \\ Hematology, Hospital of Lorient; ${ }^{5}$ Division of Thoracic and Cardiovascular Surgery, University Hospital of Rennes; \\ ${ }^{6}$ Division of Thoracic and Cardiovascular Surgery, University Hospital of Rennes; ${ }^{7}$ Division of Thoracic and \\ Cardiovascular Surgery, University Hospital of Rennes, France
}

\begin{abstract}
The incidence rate of tuberculosis in developed countries is low. The most common presentation of this disease is its pulmonary form but with the increasing use of immunosuppressive drugs, extra-pulmonary tuberculosis is re-emerging. Nevertheless, sternal bone involvement is uncommon. We report the case of an eighty-three-year-old man who presented a painful sternal mass
\end{abstract}

\section{Correspondence: Morgan Daniel, Division of Thoracic and Cardiovascular Surgery, University Hospital of Rennes, 2 rue Henri Le Guilloux, 35033 Rennes, France. \\ Tel. +33.6.83373654. E-mail: morgan.daniel@chu-rennes.fr}

Key words: Bone tuberculosis; pulmonary tuberculosis; chest wall tumor; Mycobacterium tuberculosis; thoracic surgery.

Ethics approval and consent to participate: No Ethical Committee approval was required for this case report by the Department, because this article does not contain any studies with human participants or animals.

Conflict of interest: The authors declare that they have no competing interests, and all authors confirm accuracy.

Contributions: All the authors made a substantive intellectual contribution, read and approved the final version of the manuscript and agreed to be accountable for all aspects of the work.

Received for publication: 6 November 2021.

Accepted for publication: 12 January 2022.

Publisher's note: All claims expressed in this article are solely those of the authors and do not necessarily represent those of their affiliated organizations, or those of the publisher, the editors and the reviewers. Any product that may be evaluated in this article or claim that may be made by its manufacturer is not guaranteed or endorsed by the publisher.

${ }^{\circ}$ Copyright: the Author(s), 2022

Licensee PAGEPress, Italy

Monaldi Archives for Chest Disease 2022; 92:2144

doi: 10.4081/monaldi.2022.2144

This article is distributed under the terms of the Creative Commons Attribution-NonCommercial International License (CC BY-NC 4.0) which permits any noncommercial use, distribution, and reproduction in any medium, provided the original author(s) and source are credited. which progressed towards cutaneous ulceration. The first diagnostic hypothesis was neoplasia. The pathological and microbiological diagnosis of tuberculosis was achieved after surgical biopsy. The patient received treatment against tuberculosis for nine months enabling recovery without surgery. This case illustrates the importance of having a diagnosis prior to any kind of treatment facing any voluminous parietal thoracic lesions. This diagnosis is made possible by surgical samples and interdisciplinary teamwork. This case underlines that tuberculosis remains a differential diagnosis that must be evoked in case of unusual bone mass.

\section{Introduction}

In developed countries the incidence rate of tuberculosis is low. The most common form is pulmonary tuberculosis. However, extra-pulmonary forms are not uncommon (15\%) [1], like lymph node tuberculosis or bone tuberculosis [2]. Frequency of these forms increases with immunocompromised patients [1] and at either end of the age scale. Among bone involvement, sternal tuberculosis is exceptional (less than 1\%) [2].

\section{Case Report}

An eighty-three-year-old man presented a painful sternal mass which progressed towards cutaneous ulceration associated with fever and asthenia. That sternal lesion was growing for five months (Figure 1). His past medical history included Bowen's disease, polycythemia vera and arterial hypertension. His medication included ruloxitinib and acyclovir. The past treatments received for polycythemia vera were hydroxycarbamide, interferon, pipobroman and ruloxitinib. Associated to these clinical observations the patient showed biological inflammatory syndrome (CRP $34 \mathrm{mg} / \mathrm{L})$ and anemia $(10.1 \mathrm{gr} / \mathrm{dL})$. The chest computed tomography (CT) revealed a sternal lesion of $82 \times 68 \times 57$ millimeters wide, associated with bone lysis. The CT also showed pulmonary micronodules (Figure 2).

The first hypothesis was neoplasia. A first CT-guided (11gauge needle) sternal biopsy was performed with the help of a radiologist. The sample showed no evidence for epithelial or connective tumoral process and no plasma cells proliferation. The analysis revealed granulomatous tissue without caseous necrosis.

In order to further refine the diagnosis, we decided to organize 
surgical biopsies with the help of thoracic surgery. Analysis of these new samples allowed to diagnose sternal bone tuberculosis. Indeed, the histological analysis put in evidence epithelioid granulomas with multinucleated giant cells. Ziehl coloration was negative. Direct examination (microbiological analysis) was negative but subsequent analysis with liquid culture medium was positive at day twelve, identifying Mycobacterium tuberculosis rpoB wildtype, sensitive to first-line anti-tuberculosis drugs. PCR analysis also confirmed the previous result. However, sputum examinations remained negative (direct and prolonged cultures).

A four-drug regimen against tuberculosis was initiated, including rifampicin, isoniazid, pyrazinamide, and ethambutol. Ethambutol was later stopped when the antibiogram became available. Initial treatment stage lasted three months and then the patient followed a maintenance treatment of six months (isoniazid and rifampicin). The total duration of treatment was nine months. To help healing of the deep lesion, the patient beneficiated from a dressing protocol. Full recovery was obtained (Figure 3 ).

\section{Discussion}

In developed countries, tuberculosis is rare and sternal tuberculosis is uncommon, thus explaining why our first hypothesis was

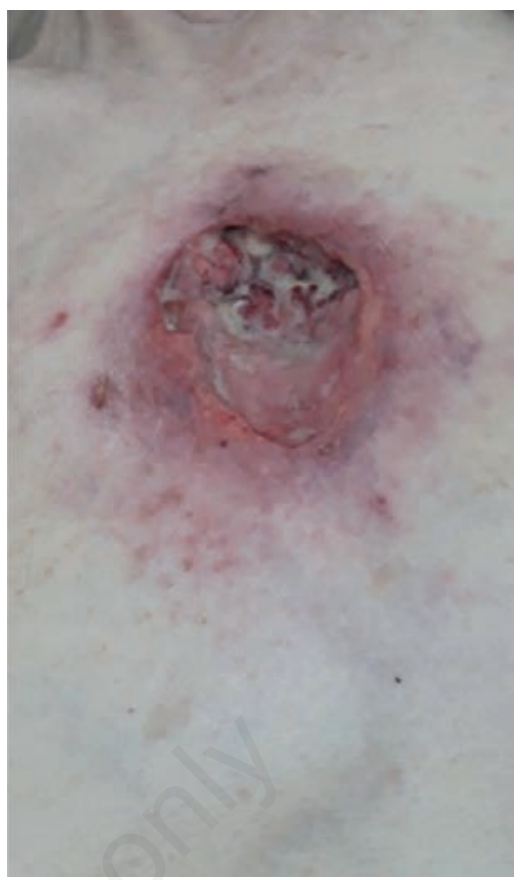

Figure 1. Aspect of the lesion at diagnosis.
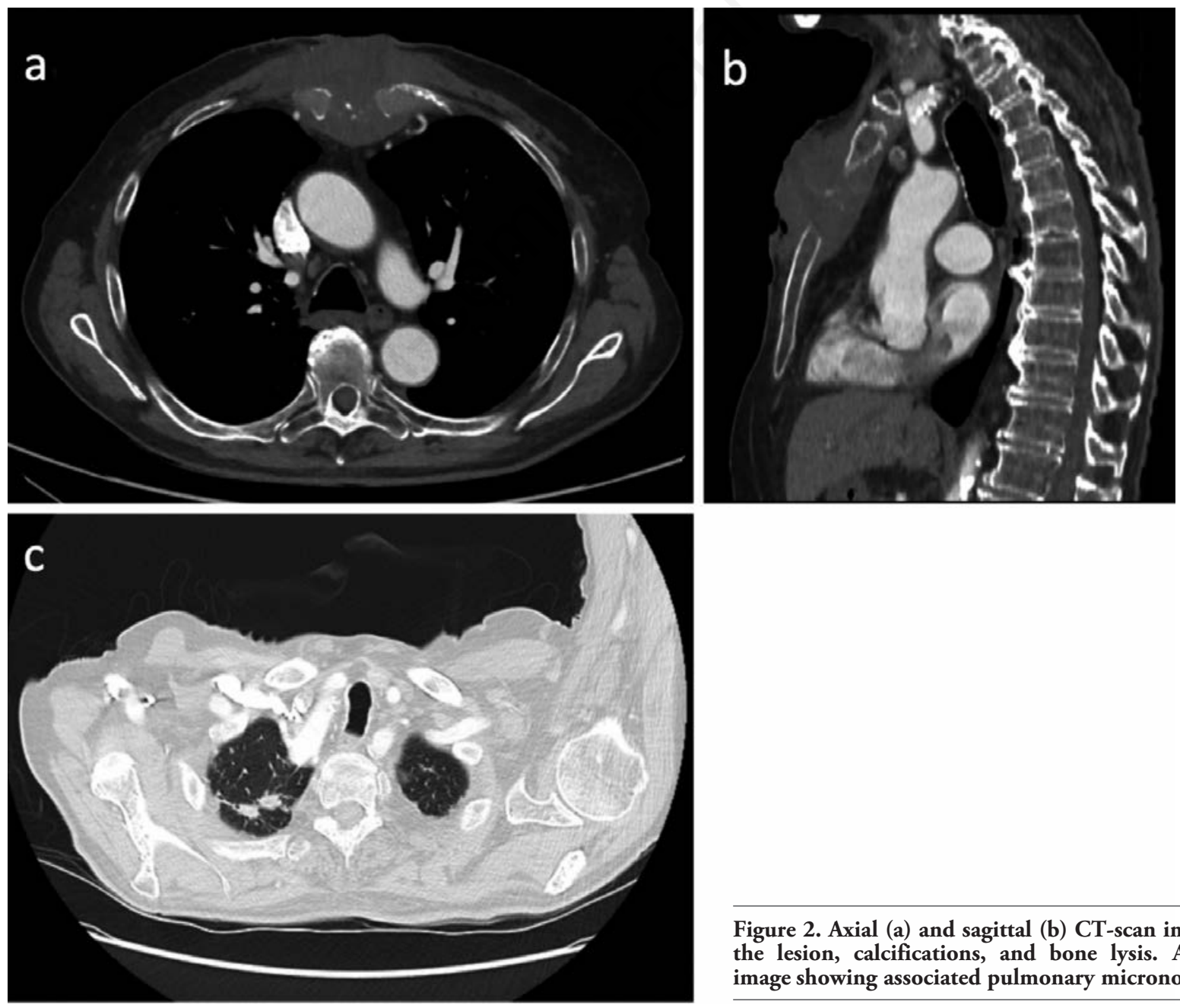

Figure 2. Axial (a) and sagittal (b) CT-scan images showing the lesion, calcifications, and bone lysis. Axial CT-scan image showing associated pulmonary micronodules (c). 

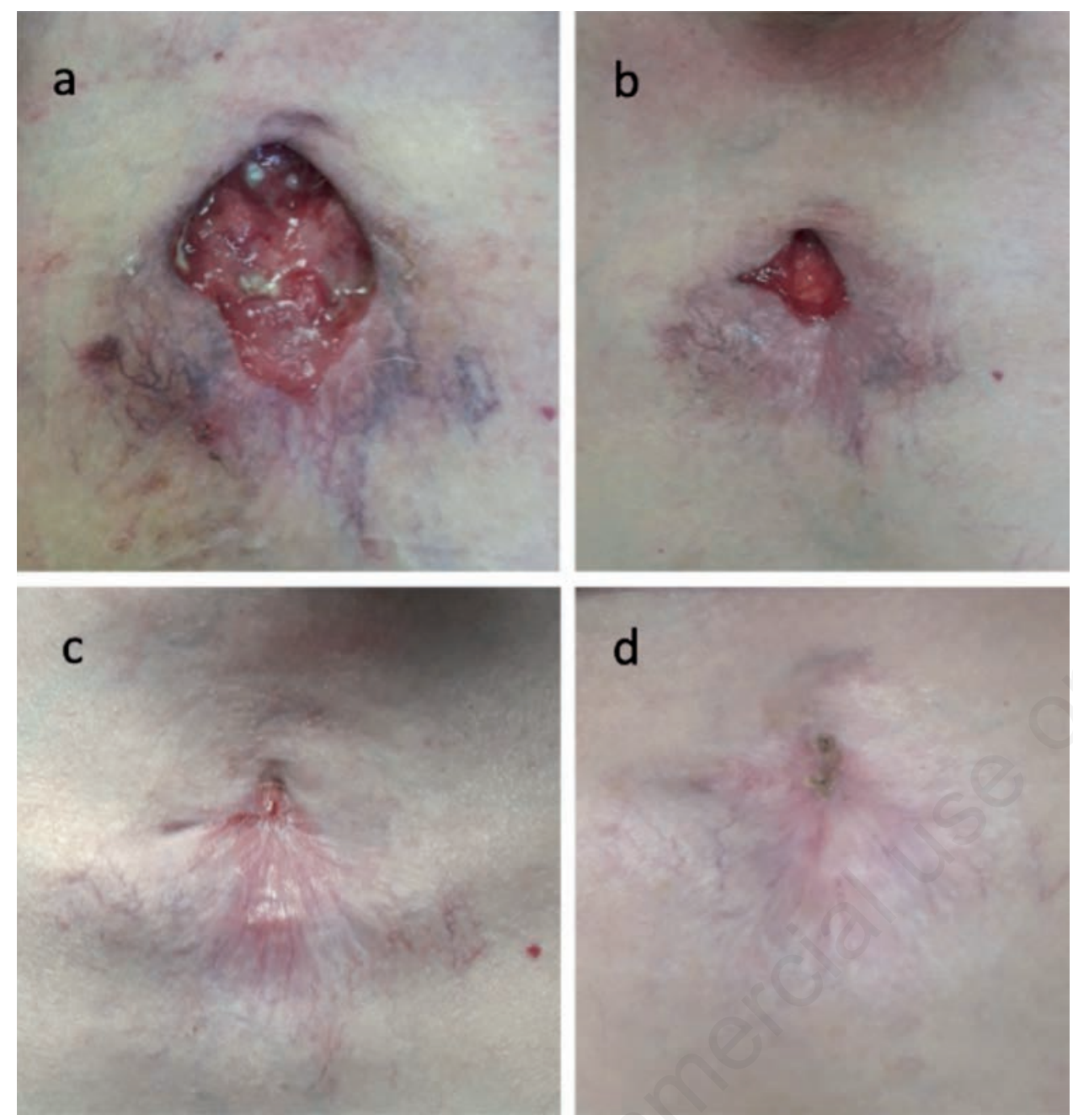

Figure 3. Evolution during treatment. Week 3 (a), month 3 (b), month 6 (c) and complete healing of the wound $(d)$.

neoplasia. Indeed, this type of lesion can be seen in primitive bone tumor such as chondrosarcoma or plasmacytoma [3]. Hypothesis of a secondary lesion was also considered, like bone metastasis of lung cancer for example.

CT guided biopsy was not sufficient to obtain the diagnosis, therefore thoracic surgery was necessary. Diagnosis was made thanks to surgical deep samples. It is mandatory when facing large parietal lesions to have a diagnosis before an eventual resection. Indeed, like in the present case, infectious etiology is always a possible diagnosis. Additionally, thoracic surgeon checklist must evoke tuberculosis even if it is not the first hypothesis: every parietal and parenchymal surgical resections undertaken in our center beneficiate from a systematic tuberculosis screening. This precautionary principle is necessary, knowing the major difference in term of prognosis and treatment between infection and neoplasia. Besides, large parietal malign lesions require histological diagnosis prior to resection surgery: margins are guided by the type of lesions and are crucial notably with sarcomas. One challenge for parietal lesions is to give patients the best outcomes while avoiding incorrect indications.

This case, beyond the fact that sternal tuberculosis is exceptional, underlines the need of multidisciplinary work (infectiologists, pneumologists, thoracic surgeons, histologists, microbiologists).

Tuberculosis is a complex systemic infectious disease, still present in developed countries. The ageing of population and the increase of treatments weakening the immune system (immunosuppressive drugs, immunomodulators, biotherapies, chemotherapies) are two important factors leading to the potential resurgence of tuberculosis [4]. Moreover, these treatments might favor extrapulmonary tuberculosis, which are more challenging to diagnose and treat.

\section{References}

1. Dheda K, Barry CE, Maartens G. Tuberculosis. Lancet 2016;387:1211-26.

2. Feki W, Ketata W, Mkaouar N, et al. [Tuberculose sternale isolée chez l'adulte immunocompétent].[Article in French with English Abstract]. Rev Pneumol Clin 2018;74:96-9.

3. Calabrò E, Pastorino U. Primary sternal tuberculosis mimicking a lytic bone tumor lesion. Monaldi Arch Chest Dis 2018;88:931.

4. Hasan T, Au E, Chen S, et al. Screening and prevention for latent tuberculosis in immunosuppressed patients at risk for tuberculosis: a systematic review of clinical practice guidelines. BMJ Open 2018;8:e022445. 